Pbilosophia Philosophia Scientiæ

Scientia

Travaux d'histoire et de philosophie des sciences

CS $7 \mid 2007$

Louis Rougier : vie et œuvre d'un philosophe engagé

\title{
Rougier et le problème de la cohésion sociale
}

\section{Thierry Leterre}

\section{(2) OpenEdition}

\section{Journals}

Édition électronique

URL : http://journals.openedition.org/philosophiascientiae/439

DOI : 10.4000/philosophiascientiae.439

ISSN : 1775-4283

Éditeur

Éditions Kimé

Édition imprimée

Date de publication : 1 janvier 2007

Pagination : 161-180

ISBN : 978-2-84174-412-1

ISSN : $1281-2463$

Référence électronique

Thierry Leterre, « Rougier et le problème de la cohésion sociale », Philosophia Scientiæ [En ligne], CS 7| 2007, mis en ligne le 08 juin 2011, consulté le 21 janvier 2021. URL : http://journals.openedition.org/ philosophiascientiae/439; DOI : https://doi.org/10.4000/philosophiascientiae.439 


\title{
Rougier et le problème de la cohésion sociale
}

\author{
Thierry Leterre \\ Université de Versailles — Saint-Quentin-en-Yvelines, \\ France
}

[...] la politique est affaire, non de parti, mais de méthode Rougier

L'historien des idées politiques, à la différence du philosophe peut-être, est condamné aux questions mal posées. Les femmes et les hommes pris dans l'action collective, la conquête ou la défense du pouvoir ou de ses formes légitimes, ne s'embarrassent pas toujours d'une expression précise de leurs opinions, de leur volonté, ou de ce qui constitue les plus objectives de leurs motivations. Et même lorsque les acteurs politiques témoignent d'un souci de vérité ou de lucidité, celui-ci se trouve subordonné à l'efficacité dans l'ordre d'action qu'ils ont choisi ou aux buts qu'ils se proposent d'atteindre. Pour autant les représentations, et notamment les représentations prétendant à la vérité, voire à la vérité universelle, ne disparaissent pas dans l'ordre politique. Tout au contraire : la prétention à dire le vrai, et à agir politiquement en fonction de cette idée de vérité en fait partie intégrante. Les questions y sont mal posées, parce qu'elles ne peuvent se régler comme des questions auxquelles il faudrait répondre d'abord en fonction de la vérité, ce qui conduirait au travail philosophique de leur élucidation. Mais enfin, elles se trouvent posées. En dehors de cette complicité du discours, nous ne rencontrons que l'instinct ou le sociologique; l'être animal ou l'être social. Non l'être politique.

Nul sans doute, plus que Rougier, n'a eu conscience de cette dualité étonnante : la revendication d'une vérité en vue de la politique, l'incapacité à assumer ce que toute forme de vérité exige de nous. Une part essentielle de sa réflexion politique se fonde sur le constat que la vérité 
constitue un enjeu de l'action collective, mais que les démarches intellectuelles propres à la découverte, ou au refus, des idées qui aspirent à posséder cette caractéristique d'être vraies, en sont tout simplement proscrites. Telle est la signification du thème, récurrent, des mystiques. Une mystique, pour Rougier, c'est un ensemble de croyances prétendant à la vérité, dérivé de doctrines argumentatives, mais pour lesquelles on a renoncé à argumenter, et de l'expérience historique dont elle finit par diverger.

A la lumière de cette analyse, on peut aborder l'intérêt spécifique qu'il y a à saisir l'œuvre politique de Rougier à partir du problème de la cohésion sociale. Cette dernière expression renvoie au fait qu'une société suppose des croyances et des attitudes partagées, pour pouvoir «tenir ensemble ». Ce n'est pas pour autant une expression conceptuelle - ni d'ailleurs une expression typique de Rougier chez qui on ne la rencontre pas : il s'agit plutôt d'une thématique qui traîne dans le discours politique contemporain. C'est à vrai dire, sinon une mystique, du moins une question mal posée et ce qui intéresse ici, ce n'est donc pas « la cohésion sociale » chez Rougier - qui ne serait rien autre chose qu'une enquête thématique sur un fantôme - mais le problème que ce que nous pouvons appeler « cohésion sociale » constitue à ses yeux. Trois développements permettent alors de rendre compte de ce problème : la question des élites, et l'opposition à la souveraineté populaire, mais aussi au gaullisme, qu'elle suppose, celle de l'État de droit, dont Rougier est l'un des plus brillants penseurs, et surtout le marché dont il présente une interprétation originale comme processus de constitution d'une société ordonnée.

\section{Une question mal posée, la « cohésion sociale»}

L'expression 《cohésion sociale » a été mise en circulation dans le domaine conceptuel par Mauss, étudiant les sociétés premières. Mais dans l'usage courant, elle appartient moins au répertoire prestigieux des concepts de l'anthropologie qu'à la famille honteuse des notions sans grande valeur philosophique. «Cohésion sociale» c'est un nom de ministère, ou de loi, dans le répertoire discursif de la politique française moderne où elle reçoit la fonction assez précise de garantir le souci social de majorités conservatrices, qu'on pourrait soupçonner d'indifférence au sort des plus démunis, sans pour autant souscrire à une idéologie « de gauche », qui, pour sa part, joue de préférence avec l'idée de « solidarité». Plus généralement, l'expression «cohésion sociale indique la nécessité 
de rassembler une société autour de valeurs fondamentales, à un moment où elle semble se disperser.

Dans ces conditions, la valeur philosophique de la «cohésion sociale» est inversement proportionnelle à sa symbolique politique. Cain de la philosophie, maudit par les dieux du raisonnement, qui favorisent les Abel des propositions bien faites. Cain maladroit au demeurant, quand il s'agit de Rougier : l'expression semble redoubler d'invalidité puisque nous ne trouvons pas sous son abondante plume l'expression ni même un équivalent approximatif. Question faible, question étrangère à Rougier : il y a quelque entêtement à vouloir se la poser.

À tout le moins, une telle obstination n'est pas tout à fait infidèle à Rougier, philosophe têtu s'il en est. Surtout, le fait qu'elle ait fini par constituer un répertoire idéologique à partir d'une importation des sciences sociales recoupe l'un des sens que Rougier assigne aux «mystiques » : une théorie argumentative et fondée en expérience (du moins pour Mauss) est devenue une croyance de nature politique. Au demeurant, tant son caractère vague que le fait qu'elle ne se trouve pas formulée comme telle chez Rougier ne doivent pas être regardés comme des obstacles. Rougier n'hésite pas à recourir à des notions larges, pour ne pas dire «molles », et à leur donner une nouvelle configuration afin de caractériser les mouvements d'idées et les attitudes sociales qui en découlent. La plus célèbre illustration de cette tendance est la notion de «rationalisme ». A peu près aussi approximative dans l'ordre philosophique que celle de «cohésion sociale» dans le domaine politique moderne, elle ne lui sert pas moins d'appui pour cerner à partir d'une optique unique, diverses dimensions de la démocratie moderne. Elle apparaît dans une grande extension, incluant jusqu'à l'empirisme dont la caractéristique récurrente était de se distinguer du rationalisme. Inversement, Rougier sépare du libéralisme, en un mouvement remarquable, l'idéologie du «laisser-faire », dont il montre qu'il s'agit d'une mystique économique juste propre, dans l'état moderne de l'économie de marché, à favoriser des trusts et des holdings. Ainsi, l'œuvre de Rougier nous montre que des notions molles peuvent aider à construire des interrogations rigoureuses.

Il n'est donc pas question de prétendre que nous allons rencontrer le problème de la «cohésion sociale » sous cette forme chez Rougier, et il n'en est pas même besoin. Simplement, on peut souligner qu'il n'est pas infidèle de soulever une telle interrogation, même mal posée, et surtout, on peut en spécifier l'utilité : poser une question mal formée à un philosophe, c'est entrer dans une certaine dimension de son interprétation en soulevant un problème dont on pense que son œuvre peut l'éclairer 
en le reformulant grâce à cette entreprise même. En ce sens, le mérite des questions mal posées, consiste à susciter l'effort philosophique de l'intelligence pour les remettre à l'endroit. Ainsi, il ne s'agit pas ici de prétendre que la «cohésion sociale » fut un problème soulevé comme tel par Rougier, mais de proposer à partir de lui une intelligence de l'œuvre de Rougier qui contribue à le formuler et peut-être à le résoudre.

\section{La question des élites}

Au plus simple, mais aussi au plus sulfureux, nous pouvons lire une préoccupation chez Rougier qui s'apparente à la question de la «cohésion sociale» dans son opuscule polémique De Gaulle contre de Gaulle. C'est un des ouvrages de polémique du philosophe, et dans son contexte personnel particulièrement, à un moment de grande tension, puisqu'il doit subir les affres de l'épuration, de retour des États-Unis. L'ouvrage a toutefois une portée plus large qu'un simple plaidoyer en auto-justification. On est juste après la guerre, à une époque de dispersion et de déchirure nationale, et de plus dans un contexte de ruine économique qui rend nécessaire un effort collectif de redressement. Tous en sont conscients, et Rougier le premier. Mais il pense que cette ardente obligation risque de se voir compromise par le jeu politique de De Gaulle, critique d'une IVe République naissante, trop faible d'après Rougier pour se permettre de telles dissensions. Incontestablement, il appartient à la famille libérale des anti-gaullistes, et il ne démentira jamais son hostilité foncière au général aventureux de 1940. L'équité veut d'ailleurs que l'on admette que les milieux proches de la France Libre ont voué à Rougier une animosité qui se traduit par sa proscription de l'université et sa mise en retraite d'office - avant qu'il ne se voie, des années plus tard, innocenté des chefs d'accusation qui avaient pu peser sur lui. Néanmoins, les sources de l'antigaullisme de Rougier sont d'abord philosophiques, et ce n'est qu'en surcharge qu'on peut discerner un mixte de positions personnelles, de justification de Vichy — quoiqu'il n'ait été Vichyssois ( «Vichyste»si l'on veut utiliser le jargon contemporain) que dans une phase très initiale du régime, quelques mois à peine, le temps d'une mission en Angleterre, avant son exil aux États-Unis - et de considérations de circonstance.

En fait, l'une des clefs de son répertoire anti-gaulliste, tient à un thème qui joue un rôle considérable dans l'œuvre politique de Rougier, la question des élites. À de Gaulle en effet, Rougier reproche ce qu'il appelle le « résistantialisme », qu'il qualifie comme l'idéologie « détruisant toute idée d'obéissance hiérarchique» [Rougier 1948b, 21]. Pour lui, c'est une 
attitude qui atteint le socle vital de ce que représente une société et en assure le maintien à ses yeux, car depuis l'avant-guerre, il n'a eu de cesse de rappeler la nécessité d'une organisation sociale où domineraient les autorités « d'élites » efficaces. Le phénomène de l'élite est même promu par lui au rang de phénomène fondamental de l'existence sociale :

«L'histoire humaine se réduit au perpétuel mouvement d'ascension, de stabilisation, de décadence et de renouvellement d'élites successives qui émergent des couches inférieures et retournent s'y absorber à leur déclin. » [Rougier 1920b, 50] « L'enseignement des sciences sociales, l'extension progressive des attributions de l'État, montrent la nécessité de déléguer à des organes différenciés, à des minorités compétentes, le travail législatif, le pouvoir exécutif et judiciaire.» [Rougier 1920b, 51]

Philosophiquement, on reconnaît la trace des thèses de Pareto, que Rougier a lu avec soin et admiration, ce qui n'est pas si commun en France à l'époque. Idéologiquement, on distingue sans mal dans une telle remarque - et dans les nombreux passages de Rougier qui vont dans le même sens - l'orientation conservatrice de son libéralisme, très hostile par exemple au Front Populaire avant guerre. ${ }^{1}$ On peut du reste admettre que l'importance de la thématique des élites explique pour une part la séduction qu'a pu éventuellement exercer sur son esprit le pétainisme. Mais c'est bien au-delà de l'idéologie qu'il faut saisir sa position, dans une analyse serrée des enjeux de toute forme de gouvernement qui amène à distinguer très clairement le rôle du pouvoir comme une forme nécessairement aristocratique de hiérarchie sociale :

«L'art de gouverner implique une sagesse, une technique et une noblesse. Il implique la connaissance du passé, la préoccupation de l'avenir, le sens des possibilités, la connaissance des moyens propres à la réaliser, le sentiment des responsabilités, et le souci des compétences. L'art de gouverner est par suite éminemment aristocratique et ne peut être exercé que par des élites. » [Rougier 1938a, 188]

Ce constat l'amène à vouloir organiser « à côté du suffrage universel » la « représentation des Corps sociaux, la représentation des compétences

\footnotetext{
${ }^{1}$ Voir par exemple Retour au libéralisme [Rougier 1938a, 186] : « l'expérience en France du Front Populaire montre [...] comment, avec les meilleures intentions du monde, on peut s'orienter vers un régime totalitaire dans le cadre constitutionnel d'une démocratie libérale. » (Il est à noter que Retour au libéralisme est largement repris dans le chapitre 1 de Les mystiques économiques [Rougier 1938b]).
} 
à côté de celle des partis » [Rougier 1929b, 9]. Il prend soin de préciser que cette affirmation du rôle des élites n'est pas anti-républicaine d'essence : c'est bien en plus et non à la place du suffrage universel ${ }^{2}$ qu'il souhaite imposer une représentation des élites comme un correctif à l'impossible «compétence de la majorité » [Rougier 1920b, 30]. Il s'agit là d'une réorganisation de la démocratie, ou plutôt d'une certaine démocratie, celle qu'il a sous les yeux, mais non de sa suppression, ni même de sa subversion. A aucun moment Rougier n'est tenté d'en finir avec la démocratie, ${ }^{3}$ et c'est d'ailleurs ce qui l'amène à établir les bases d'un élitisme républicain fondé sur un recrutement des élites ouvert à toutes les classes sociales. L'affirmation ne possède pas qu'un sens fonctionnel, au sens où elle toucherait exclusivement à l'organisation des institutions et du régime. Bien plus fondamentalement, elle coïncide avec une propriété essentielle de la démocratie caractérisée précisément par « le libre recrutement de l'élite dans toutes les classes de citoyens. » [Rougier 1927a, 325] Ainsi se dessine la bonne configuration de la cohésion sociale en régime démocratique selon Rougier : des élites largement recrutées, soumettant à leur autorité un peuple qui bénéficie d'une bonne gestion des affaires publiques favorisant une économie dynamique.

\section{État 《 démo-libéral » et refus de la souverai- neté populaire}

Par sa théorie de l'élite démocratique, Rougier peut s'assurer que la démocratie ne déroge pas à la condition de toute forme politique, la mise en place d'un système essentiellement aristocratique de gouvernement. Il n'en reste pas moins fidèle à l'étymologie du terme « démocratie ». Le démos a sa place marquée dans les conceptions de Rougier : il devient un véritable réservoir à élite, et probablement le fonds de toute élite

\footnotetext{
${ }^{2}$ Ici la distance par rapport à ce qui est - dans une chronologie postérieure au texte mentionné - devenue l'idéologie de Vichy est patente.

${ }^{3} \mathrm{Il}$ arrive à Rougier d'évoquer des moyens « anti-démocratiques» pour brider la démocratie parlementaire. Mais il évoque à ce titre le «bicaméralisme » et l'État de droit, sur lequel on reviendra. Ni l'un ni l'autre de ces mécanismes institutionnels ne nous paraissent aujourd'hui scandaleusement démocraticides (même s'il reste des fractions, désormais marginales, de l'opinion pour les critiquer). Il ajoute le «suffrage censitaire » qu'il repousse car «trop voyant ». Cela signifie qu'il n'y a pas d'attachement de principe à la démocratie, mais un constat de réalisme : la démocratie est le régime qui convient pragmatiquement à une société moderne. On peut être surpris d'une telle affirmation, mais elle est parfaitement homogène à la position par exemple d'un Chuchill, autre libéral conservateur, lorsqu'il explique, dans une phrase célèbre que « la démocratie est le pire des régimes à l'exception de tous les autres ».
} 
moderne. En ce sens, Rougier parle d'un « État démo-libéral » [Rougier 1938b, 35] qui à ses yeux représente un équilibre dont on peut penser qu'il est optimal. Si l'histoire politique de tous temps est celle de l'ascension et du déclin des élites dans la hiérarchie sociale, le fait de puiser des ressources dans toutes les couches de la hiérarchie sociale assure une pérennité optimale de la circulation des dominants. N'excluant personne a priori, le mécanisme peut intégrer tout le monde et réduire par là les conflits de classe en vue de la conquête du pouvoir.

Cela, il est vrai, Rougier ne le dit pas, et l'on voit bien pourquoi : une telle affirmation conduirait à ouvrir vers la gauche un système qui demeure indéniablement conservateur. En revanche, la pensée politique de Rougier se développe au moins dans deux directions compatibles avec cette affirmation qui demeure implicite. La première est essentiellement économique et constitue une légitimation démocratique du marché : l'augmentation du niveau de vie, du bien-être général, est un des buts principaux d'une politique moderne. Tous, même si c'est à des degrés divers, et même comme l'avoue crûment Rougier, de manière inégale, participent des bienfaits d'une économie performante. Rien ne permet de soupçonner chez lui cet attachement sincère à la cause d'un progrès matériel partagé par tous à la racine de sa justification, d'ailleurs classique, d'un système de marché permettant d'atteindre un niveau de prospérité matérielle auquel aucune autre organisation ne peut prétendre.

Une seconde dimension de la réflexion sur la démocratie renvoie à sa définition même, car Rougier insiste sur le fait que la démocratie doit être considérée comme le régime visant le bien du plus grand nombre. Cette conception transforme le rôle que peut jouer le peuple dans l'organisation des pouvoirs. Il est le destinataire de l'action du gouvernement, mais, et c'est un point crucial aux yeux de Rougier, il ne saurait être, sans contredire à la règle élitaire qui préside à sa politique, la source de ce pouvoir. De telle sorte, la démocratie se voit considérée non comme le régime où tous décident mais comme celui où la grande majorité prospère grâce à l'action d'élites efficaces — gouvernement du peuple, pour le peuple, mais pas par le peuple, pourrait-on dire. En insistant sur cette position, Rougier recourt à une double opération. Tout d'abord il renoue avec une définition classique, antique même de la démocratie, puisqu'il ne cite pas moins que Périclès, au début de La mystique démocratique pour appuyer son propos. Ensuite, il envisage les conséquences critiques de ce développement qui l'amènent à récuser l'idéologie de la «souveraineté du peuple.»

La référence à Périclès possède plusieurs sens sous la plume de Rougier, lorsqu'il la mobilise dans La mystique démocratique. Le premier, 
qui est loin d'être accessoire, est de témoigner de la permanence d'une culture classique au sein d'une analyse contemporaine du fait démocratique. Elle illustre parfaitement la dualité de la «connaissance du passé » et de la «préoccupation de l'avenir » qui définit la sagesse aristocratique du gouvernant et traduit aussi le souci de faire sa place à la culture au sens traditionnel du terme - l'étude des anciens, des langues mortes, de la littérature. En un autre sens, cette référence manifeste un souci de la culture représentant une nuance dans le système libéral que Rougier préconise. En effet, dans cet « État démo-libéral » gouverné par l'ambition de la prospérité économique, le risque est présent de conduire à l'élimination de toute autre préoccupation que celle de l'aisance matérielle. C'est pourquoi La mystique démocratique ne cesse d'affirmer la valeur d'une élite cultivée, soucieuse non seulement de l'histoire sous la forme du développement économique, mais également témoin d'une culture esthétique classique. C'est une conséquence qui semblera surprenante, mais Rougier accepte ce qu'entraîne le critère de la prospérité, admettant implicitement que ce qui ne va pas paraître utile risque tout simplement de disparaître. C'est pourquoi, dans un pessimisme qui a été celui de bien des intellectuels libéraux qui lui sont contemporains (on pense à Arendt, à Schumpeter, dont Rougier est par ailleurs très proche intellectuellement) il conclut que dans ce monde dont il mesure la hiérarchie des compétences, c'est tout simplement la culture qui est menacée, bien plus qu'une cohésion sociale qui rend solidaires le prolétaire et le capitaliste. $^{4}$

Parler de Périclès, permet donc d'illustrer la contribution d'une culture classique à la pensée politique, et de donner une image concrète de cette « connaissance du passé » qui fait partie de l'art de gouverner. Mais cette référence a un autre sens encore : elle souligne par contraste le caractère récent et de nature révolutionnaire de la conception « canonique » de la démocratie, fondée sur une participation directe du peuple aux affaires, sur laquelle Rougier se concentre à partir d'une critique fondamentale de la souveraineté populaire. En effet, le thème des «compétences» signifie le rejet de ce « dogme de la souveraineté nationale [qui] [...] est l'une des conceptions métaphysiques les plus erronées que nous ait léguées l'idéologie révolutionnaire ». Ce refus s'entend en deux sens au moins. D'une part, le peuple comme collectif, n'est pas un souverain, au sens où il n'est pas une entité dotée d'une personnalité politique éminente. Ce

\footnotetext{
${ }^{4}$ « la véritable classe déshéritée, dans notre monde où prédominent les intérêts économiques, est celle des intellectuels » [Rougier 1929b, 10]; cf. également (mais au style direct d'apostrophe, qui introduit une nuance rhétorique) : « Travail et capital ne s'opposent pas : il sont partie liée car les hauts salaires dépendent des super bénéfices [...] cependant qu'à pas lents s'exile du monde le cortège des muses. »
} 
n'est qu'une masse inorganique; rien de défini n'en saurait émerger : « la volonté des gouvernés en tant que générale n'existe pas, qui est variable, capricieuse [...] incohérente, contradictoire [...]» [Rougier 1920b, 51]. D'autre part, et en un sens plus particulièrement politique, le refus de la souveraineté signifie le déni de la compétence politique de la majorité. Celle-ci n'est pas capable de prendre les bonnes décisions car elle manque des connaissances, de l'éducation et même du jugement nécessaires.

On peut reconnaître en cela l'héritage du libéralisme classique, celui de Montesquieu par exemple, lorsqu'il affirme que le peuple ne peut pas décider, mais qu'il peut désigner ceux qui gouverneront. Encore, chez Montesquieu il s'agit d'intégrer le peuple dans le jeu politique, en dépit de son incompétence foncière pour les affaires du pouvoir. Chez Rougier, la même idée s'infléchit au contraire sur une hostilité non dissimulée à l'égard des mouvements populaires. L'analyse rend des accents particulièrement sombres lorsque Rougier s'exprime sur la masse, ce peuple « toujours prêt à assister le crime heureux et à bafouer la vertu qui ne rencontre pas sa chance $»$.

« la masse est futuriste : elle fait table rase du passé, tient les formalités, la politesse, les égards, les traditions, le respect des règles du jeu social pour d'inutiles complications $» .^{5}$

L'affirmation du rôle des élites relève alors de la même logique que la méfiance à l'égard du peuple : la majorité ne peut pas assumer les fonctions nécessaires à un ordre social hiérarchisé, formel, que demande la cohésion sociale, entendue comme ensemble des rapports réglés au sein d'une collectivité.

\section{Démocratie, inégalité et État de droit}

Chez Rougier, la récusation de la souveraineté populaire se développe dans trois dimensions. Tout d'abord, il y a une méfiance immédiate à l'égard de la masse; ensuite une volonté de hiérarchie sociale en fonction des compétences. L'une et l'autre s'articulent à la même conception du paralogisme que constitue la domination du grand nombre des incompétents, sur le petit nombre de l'élite. Ce constat amène le troisième plan de la réflexion de Rougier : l'affirmation de l'inégalité entre les hommes et entre les peuples. C'est une erreur que de croire que «non seulement tous les individus sont égaux, en vertu de la loi naturelle, mais [que] tous

\footnotetext{
${ }^{5}$ [Rougier 1938a, 189] (texte repris dans [Rougier 1938b]).
} 
les peuples le sont aussi. » Rougier prend parfois des accents nietzschéens pour dénoncer l'idéal égalitaire de la «mystique démocratique. »

«L'égalité est toujours restrictive de la liberté des forts ou des privilégiés au bénéfice des faibles et déshérités. » [Rougier 1929b, 38]

Rien ne vient corriger cette distorsion car Rougier écarte même la solution, désormais classique, de l'éducation de la masse, censée diffuser les connaissances et donc éclairer le jugement des citoyens. À ses yeux, le développement des systèmes scolaires ne représente pas une œuvre de culture, mais une étatisation d'un domaine, l'éducation, qui devrait rester privé et l'ultime combat d'un rationalisme qui s'épuise à corriger des différences irréductibles en prétendant que si les hommes sont égaux, alors seule la différence des éducations peut expliquer ce qui reste de dissemblance entre eux. ${ }^{6}$

L'affirmation semble brutale et elle l'est en effet : Rougier est l'un des rares penseurs de l'inégalité dans les temps modernes. Pourtant, il ne faut pas lui faire dire plus que ce qu'elle ne dit en effet. Tout d'abord, ce refus de la masse le prémunit contre l'illusion totalitaire qu'il dénonce très rapidement, passé une hésitation initiale sur le fascisme italien. Rougier est l'un des premiers à soulever, il est vrai dans un esprit critique de la démocratie, la question de la liaison entre totalitarisme et démocratie, et à constater qu'il s'agit là d'une tyrannie d'un type spécifique qui agit sur et par les masses.

De plus, si Rougier est l'un des rares penseurs du $20^{\mathrm{e}}$ siècle à assumer une position clairement inégalitariste, ce n'est pas essentiellement en vertu d'un différencialisme qui affirmerait l'inégalité des hommes en euxmêmes. Sans aucun doute, il écrit dès Les paralogismes du rationalisme qu' « un homme n'en vaut pas un autre, quoi qu'en ait pensé Diderot » Cette différence entre les personnes n'est toutefois pas intrinsèque, mais renvoie plutôt à l'expression sociale de la diversité des talents. L'inégalité entre les hommes est en effet mesurée chez Rougier par un critère purement extérieur, la difficulté à remplacer une personne occupant une fonction :

« la valeur d'un individu, du point de vue social, dépend du degré de bienfaisance de ses actions et du degré de difficulté qu'on éprouver à le remplacer. Plus son activité est précieuse pour la collectivité, moins il est aisément remplaçable et plus

\footnotetext{
${ }^{6} \ll[\ldots]$ les différences entre [les individus et les peuples] ne peuvent plus provenir que d'une différence d'éducation » [Rougier 1920b, 22].
} 
doivent s'accroître ses fonctions et ses privilèges. » [Rougier 1920b, 43], [Rougier 1929b, 47]

L'expression « bienfaisance » ne doit pas inciter à un contresens moraliste. Rougier subvertit la terminologie éthique de la bienfaisance, en prenant le terme très précisément au sens de «ce qui fait du bien », ce qui est utile socialement. C'est pourquoi l'utilité sociale est la véritable mesure, qui permet de montrer que dans l'activité sociale, il est plus difficile de remplacer Pasteur que son garçon de laboratoire, pour emprunter à Rougier l'un de ses exemples. Le point de vue reste donc extérieur à la personne. Nous sommes inégaux en termes d'utilité sociale; en revanche, que nous sommes tous égaux en tant que titulaires de droits individuels qu'il faut absolument protéger.

C'est pourquoi, même si Rougier assume le vocabulaire de l'inégalité, il est clair qu'une partie de ce discours relève d'une confusion entre celleci et la notion de diversité. Le diagnostic est net et porte sur «la vie décolorée d'un conformisme abêtissant » qui «menace de nos jours la civilisation occidentale » [Rougier 1938b, 31] d'où le refus d'une idéologie de la justice «entendue comme uniformisation des conditions » car «les libertés individuelles » « engendrent la diversité » [Rougier 1927a, 344]. Le critère de l'utilité implique que les compétences sont aussi diversifiées que les sources de l'utilité sociale, et l'on s'explique son aversion extrême pour les aventures fascistes qui finissent par incarner le corps social dans un duce, un conductor, un Führer. Si Rougier a été inoculé contre les tentations totalitaires - et l'on doit garder à l'esprit que son soutien à Vichy est strictement conditionné au fait qu'il récuse une interprétation du régime qui en ferait un fascisme français — c'est en fonction de cette volonté de maintenir la diversité d'une société et de ses élites politiques et sociales.

Dès lors, l'éloge de l'inégalité comme diversité débouche sur une antinomie classique, celle de l'égalité et de la liberté, dont la conséquence la plus nette est que chez Rougier, l'obéissance aux autorités et aux compétences n'a rien d'absolu, et se voit limitée à la sphère du gouvernement, dont il veut que les attributions soient réduites.

« [la démocratie libérale] considère que le but de l'institution politique est de défendre, de toute la force du corps social, les libertés qui seules permettent de traiter les citoyens comme des créatures douées de libre décision et de responsabilité. Elle repose sur une déclaration des droits de l'homme et du citoyen et suppose au-dessus du pouvoir exécutif et du pouvoir législatif une instance souveraine qui frappe de nullité 
non seulement les abus administratif comme le Conseil d'État en France, mais aussi les lois qui violent les garanties constitutionnelles [...] comme aux États-Unis. » [Rougier 1963b, 256 et suiv.]

Rougier se révèle sur ce point être indissociablement un libéral et un conservateur. Le conservateur approuve l'ordre et la hiérarchie. Le libéral exige des bornes très étroites à l'étendue de leur influence. C'est ce qui l'amène, en référence à Constant, à noter que ce qui importe n'est pas 《 qui » détient le pouvoir, mais quelles sont les limites de son étendue [Rougier 1963b, 256 et suiv.]. Ce réquisit se fonde sur la discrimination axiologique de la liberté, non de l'égalité, comme valeur essentielle. Ce constat implique que l'autorité publique, si nécessaire apparaisse-t-elle, si hiérarchique et hiérarchisée doive-t-elle être dans son organisation, soit strictement encadrée en fonction d'une logique simple, classique chez les libéraux, mais comme Rougier en a conscience, relativement rare dans la pensée politique française : là où il y a des règles, y compris des règles générales, publiques, la liberté des individus risque de se voir contrainte. Si ces règles sont inspirées par un idéal égalitaire, ajoute Rougier, alors l'oppression n'est pas loin, car le jeu naturel de la liberté est de créer des inégalités.

C'est pourquoi Rougier réfléchit au sens concret que revêt la protection des libertés dans une société : s'il est crucial que la liberté soit garantie par des droits individuels absolus, strictement limitatifs de la puissance publique, il faut que ces droits, qu'on trouve dans les déclarations du 18e siècle, se voient juridiquement assurés par le biais de leur inscription au niveau constitutionnel, et par l'existence d'une cour capable d'en juger et de protéger le citoyen.

Dès lors, Rougier apparaît comme un extraordinaire analyste de l'État de droit. Il l'est dès les années 60 , dans le sillage de sa critique de la constitution de 1958, à une époque où les plus renommés des juristes français en distinguaient à peine l'organisation et n'acceptaient guère la notion même d'État de droit au sens qui est devenu classique et qui est celui de Rougier. Il faut d'ailleurs souligner la valeur de sa réflexion non seulement parce qu'elle anticipe sur la vie constitutionnelle de la France, mais encore parce que pour Rougier c'est un retournement sensible. Quand il écrit ses Paralogismes du rationalisme, les droits de l'homme ne sont que l'une de ces vaticinations mystiques léguées par l'histoire du rationalisme. À mesure, par leur inscription dans l'État de droit, ils deviennent partie prenante de la cohésion sociale d'une société libérale. 


\section{Cohésion sociale et refus du gaullisme}

Rien au fond n'est plus révélateur de la conception qu'entretient Rougier de cette cohésion sociale - si l'on désigne par là la façon dont une collectivité s'organise comme telle, dans sa singularité et dans le réseau de ses solidarités — que son opposition au gaullisme. Celle-ci n'est pas simplement le conflit d'un homme probablement humilié par deux procès en indignité nationale (qui se terminèrent toujours en le rétablissant dans ses droits). Elle est plutôt l'expression d'un génie libéral qui remet en cause les fondements mêmes de la geste gaulliste, y compris sur des points qui semblent très proches. La question de l'État de droit est révélatrice : il est ironique que l'essence même de la critique qu'adressait Rougier à la constitution de la $\mathrm{V}^{\mathrm{e}}$ République - l'absence de défense constitutionnelle des droits individuels ${ }^{7}$ - ait fini par être le pivot de notre vie constitutionnelle en 1971, quand le conseil s'opposa à Pompidou au nom des droits de l'homme rappelés dans le préambule de la constitution. Ceux-ci prirent, de ce fait, et comme le voulait Rougier, une valeur constitutionnelle. Il fallait la constitution gaulliste pour réaliser cette évolution que Rougier appelait de ses vœux en la croyant improbable, et que de Gaulle aurait vigoureusement écartée.

L'autre point de contact, mais aussi de répulsion, est incontestablement cette question des élites. Chez de Gaulle, demeure un césarisme de principe qui affirme la souveraineté populaire - que Rougier récuse et son expression dans l'homme politique, l'homme d'exception, particulièrement lorsque surgit la crise. Du côté de Rougier, les élites demeurent plurielles. Ce sont, comme il le note, des « Corps sociaux», des «organes différenciés » qui se distinguent de la masse et l'encadrent. Cette pluralité confère aux théories de Rougier un caractère organiciste où il n'est pas difficile de discerner l'idée d'un corps politique dont chacun serait l'organe et au sein duquel il trouverait sa place.

Cette place, elle est aussi bien déterminée par un système de marché dont Rougier affirme la supériorité : les «compétences» sont intimement liées au développement économique. Elles représentent à la fois les élites issues d'un jeu capitaliste qui crée des inégalités, et les spécialistes capables de prendre les mesures adaptées pour faire fonctionner le marché. C'est un autre point de divergence par rapport au gaullisme puisqu'il n'y a pas chez Rougier de vision d'une élite républicaine, vouée au service de l'État, d'un État qui prendrait une plus large part au sein de l'économie par les nationalisations, par des politiques budgétaires s'éloignant

\footnotetext{
${ }^{7}$ [Rougier 1963b, 261-262] : Rougier stigmatise l'existence de droits « qui, ne faisant pas corps avec la constitution, n'aura qu'une valeur indicative. »
} 
de l'orthodoxie ou par l'invention d'un rôle redistributeur. Nous sommes loin des technocrates de la $\mathrm{V}^{\mathrm{e}}$ République et proche d'un univers de notabilité où celle-ci ne reposerait certes plus sur les élites du passé, mais sur une bourgeoisie industrielle, capitaliste, mais aussi une bourgeoisie intellectuelle cultivée, particulièrement apte à diriger un pays dans un contexte d'économie moderne, une économie de marché.

Ce qu'on peut appeler «la cohésion sociale» chez Rougier correspond à la représentation d'un peuple divisé entre gouvernants et gouvernés, sans que l'osmose, même théorique, de la souveraineté ne vienne réduire la différence. Ces gouvernants, inégaux entre eux, et subordonnés à un principe d'autorité sont issus d'élites capitalistiques particulièrement dynamiques, ou aptes à régler le fonctionnement du capitalisme à l'horizon d'un optimum dont il emprunte, brillamment, l'idée à Pareto, à la mémoire duquel il dédie l'un de ses ouvrages. On saisit que de Gaulle ne présente de cette conception qu'une vision déformée : la distinction des gouvernants et des gouvernés se réduit dans une conception incarnative du pouvoir, la volonté d'organiser un contexte favorable au capitalisme se traduit pas une prise en charge étatisée de l'économie. Rougier peut alors souligner à l'envi les hésitations de De Gaulle devant la politique économique à mener, et ses incohérences dans De Gaulle contre de Gaulle. Il montre le Général pris entre nationalisations et éloge de l'entrepreneur, et cette tension traduit à ses yeux deux choses au moins : l'insuffisance de De Gaulle comme homme d'État moderne, chargé de reconstruire grâce aux ressources d'un marché dynamique une France ruinée, et plus fondamentalement, une vision autoritaire d'un État qui risque de dégénérer. Telle est d'ailleurs l'impitoyable diagnostic de Rougier sur la $\mathrm{V}^{\mathrm{e}}$ République : elle est bien près d'un État de police.

\section{Le marché, force de cohésion sociale}

La critique du gaullisme économique, si elle possède un sens polémique, n'en renvoie pas moins à une perspective originale chez Rougier : l'introduction d'une réflexion philosophique à partir d'une théorie du marché. Rougier a, sur ce point, conscience d'ouvrir une voie en France et il note qu'avant la première guerre mondiale, l'économie n'était pas un objet légitime pour un philosophe - on peut dire qu'en France, à l'exception de quelques ouvrages rares comme les Propos d'économique d'Alain cela a été longtemps le cas. Encore doit-on saisir que la pensée du marché chez Rougier ne se limite pas à l'économie mais représente un paramètre important de la cohésion sociale. Nous sommes très loin, chez 
lui, d'une vision atomisant l'économie de marché entre des échangistes qui se rencontreraient le temps de la fixation d'un prix et se sépareraient ensuite. Rien de moins anonyme et froid que le capitalisme selon Rougier : au contraire, l'économie est le lieu où se tissent des rapports sociaux particulièrement denses.

Cette intensité du social s'illustre à partir d'un renversement remarquable, capital même : pour Rougier, le marché opère selon une justice distributive, qui donne plus à celui qui a plus, et moins à celui qui a moins. C'est s'opposer à toute la tradition et cela jusqu'à Aristote (qui en forge les termes ${ }^{8}$ ) regardant l'échange économique comme la forme la plus évidente de justice « commutative» fondée sur l'égalité entre ce qui est donné et ce qui est reçu. Clairement pour Rougier, le capitalisme distribue des biens sociaux, autant qu'économiques, de manière inégalitaire. Le riche capitaliste obtient plus de considération, de pouvoir et d'influence, que son employé, qui en a d'autant moins. C'est en cela que le marché crée de la hiérarchie et des compétences. En cela il va s'opposer par exemple à un économiste contemporain comme Schumpeter, auquel on peut le comparer sur bien des points : même admiration pour le capitalisme de marché, même vision élitiste du politique, même désacralisation du fait démocratique et même désenchantement devant un univers dont il perçoit la médiocrité spirituelle. Pourtant Schumpeter décèle dans le marché une dynamique qui finit par se détruire socialement car elle s'avère incapable de maintenir les élites politiques traditionnelles. Le capitalisme, pour diverses raisons, programme la destruction de l'ordre social. Pour Rougier tout au contraire, il y contribue, car il distingue dans le jeu du capital une hiérarchie nouvelle intéressée à la politique. Il y a à cela une raison précise, qui tient à la théorie économique : la nécessité de l'intervention de l'État dans la régulation du marché :

«Une économie libérale implique [...] une certaine intervention de l'État [...] le dirigisme de l'État libéral implique qu'il soit exercé de manière à protéger la liberté, non à l'appauvrir. $\gg^{9}$

Pour Rougier en effet, le marché sans intervention de l'État est un mythe, une «mystique libérale», celle des physiocrates, et du « libéralisme manchestérien » qui au fond, ont reproduit dans la sphère économique les errances du rationalisme en pensant que la raison pouvait s'imposer spontanément dans le jeu économique. Pour ce motif, il rejette l'idée du « laisser faire » et montre qu'elle s'avère contre-performante.

\footnotetext{
${ }^{8}$ Éthique à Nicomaque, V,7. La traduction française classique de Tricot parle plutôt de « juste correctif » à la place de « commutatif » .

${ }^{9}$ [Rougier 1938b, 84] (souligné par l'auteur).
} 
«[...] la libre concurrence ne va pas sans malfaçons qu'un contrôle étatiste et une organisation internationale confiée à des agents compétents pourraient faire disparaître. » [Rougier 1920b, 46]

On notera la hardiesse du propos, qui n'hésite pas à admettre un contrôle international auquel Rougier s'est toujours attaché. Ce cosmopolitisme des élites est du reste partie prenante de son mépris à l'égard du peuple, qui se révèle incapable d'aller plus loin que ses intérêts bornés, qui l'attachent à un nationalisme étroit. Mais ce qui domine, à part cette reconnaissance de la nécessité d'un réglage du marché au niveau mondial - ici Rougier anticipe très correctement sur le mouvement de mondialisation qui a débouché sur l'OMC — c'est la spectaculaire reconnaissance des déviances potentielles d'un ordre de marché. Il n'y a pas de perfection du libre jeu de la concurrence. À ses yeux, une concurrence sans frein aboutit à une situation de monopole - et à la différence de Schumpeter, il n'a pas en tête, en dépit de sa vaste culture économique, la théorie de la concurrence monopolistique - qu'il rejette. L'insistance est réelle, et on le perçoit à un signe très sûr : Rougier qui se montre hostile aux mesures de Roosevelt dans la période du new deal n'en approuve pas moins les lois anti-trusts. Certes, tout comme Hayek, il pense que le jeu économique s'équilibre spontanément. Mais cette spontanéité demande un appareil de stabilisation de l'économie de plus en plus complexe. Il sait parfaitement, mieux que nombre de libéraux, que les contrats, la propriété sont des notions juridiques, non économiques, qu'elles renvoient à une construction historique, et non à des concepts naturels. Ils demandent une intervention de l'État, et Rougier le reconnaît d'autant mieux qu'il voit dans ceux qui ont la charge de cette intervention le cœur de l'élite compétente qu'il réclame.

Il ne s'agit pas pour autant d'une élite technocratique. Rougier n'hésite pas à dénoncer le «rêve des technocrates» [Rougier 1938b, 200], c'est-à-dire le rêve de gouverner politiquement l'économie par le dirigisme, où il voit les semences du totalitarisme, parce que l'État doit assumer dans le gouvernement de l'économie un rôle régulatoire, et non détenir une fonction organisatrice. En cela, il s'oppose encore à de Gaulle, pour lequel le thème des compétences, des élites, recouvre bien la construction technocratique d'un État pris dans une logique d'extension. Certes, Rougier accepte le «renforcement de l'Exécutif » [Rougier 1938a, 196]. Il le souhaite même pour lutter contre la montée en puissance des totalitarismes avant guerre. Mais il maintient toujours que ce renforcement doit s'accomplir dans une sphère de compétence limitée. En bon libéral, 
il peut accepter plus de pouvoir au pouvoir, non une administration plus étendue de ce dernier.

\section{Retour au démocrate}

On rencontre alors une distinction, implicite mais pleine de finesse, entre État technocratique et gouvernement des compétences. Elle permet de maintenir la différence entre des formes conservatrices d'organisation sociale et le risque d'un dirigisme à partir des structures politiques. Là encore le marquage libéral est sensible. Autant l'idée d'une société en bon ordre, dominée par des élites compétentes séduit Rougier, autant cette même domination, à partir du foyer politique de l'État, entraîne un risque fondateur. Politiquement, cela explique que lorsqu'il envisage concrètement la réforme de l'État à partir de cette idée d'élites compétentes, Rougier le fait en ajoutant leur représentation à celle du suffrage universel, et non en substituant - comme c'est le cas sous la $\mathrm{V}^{\mathrm{e}}$ République Française - des élites d'État au rôle de la représentation politique. Dans une version plus tardive, lorsqu'il plaide pour le développement de l'État de droit, le mécanisme est identique dans son inspiration : il s'agit de compléter le suffrage universel, non de le remplacer. Dans tous les cas, Rougier est fidèle à la formule selon laquelle il faut « amodier» la démocratie parlementaire.

L'État qui intervient dans l'économie de marché n'est donc pas pour Rougier un État qui forme en son sein des hauts fonctionnaires chargés du capitalisme. C'est plutôt un État qui coopère avec les élites de ce que nous appellerions la «société civile» et dont une partie provient de l'ascension du capitalisme. On retrouve bien sur plan ce qui constitue l'attachement fondamental, irréductible, de Rougier à une conception pluraliste, non seulement des élites, mais aussi, et de manière encore plus radicale, de l'organisation d'une société. La cohésion sociale ne saurait être l'uniformité de l'administration pas plus que le conformisme du capitalisme monopolistique. Elle doit émerger du bon ordonnancement de la société à partir de la production de ses élites. D'où la tonalité finalement démocratique des positions de Rougier — peut-être sans que lui-même en prenne conscience. Sa critique de l'État, et donc de la technocratie, repose sur l'intuition fondatrice d'une production des élites à partir du peuple ce «fonds commun d'où émergent progressivement les oligarchies qui le dominent et où elles retournent s'y dissoudre. » [Rougier 1920b, 50] L'État n'est donc pas le foyer irradiant des compétences 
nécessaires à la bonne organisation (et à la bonne hiérarchie) sociale. Il en est plutôt la caisse de résonance.

La cohésion sociale devient alors un jeu triangulaire entre l'État, le marché, les compétences qu'on peut résumer ainsi : le marché renforce l'État par l'augmentation du bien-être et produit des élites de gouvernance qui vont notamment guider l'État. Pour autant cet État ne demeure pas passif et dans un des passages les plus remarquables de finesse qui ait été consacré aux relations entre économie libérale et théorie économique, Rougier note le caractère partiel du fait économique au sein d'une conduite politique :

«Une politique absolument justifiée sur le plan économique peut cesser de l'être sur le plan sociologique, dont le facteur économique n'est qu'un des facteurs déterminants. » [Rougier 1938b, 76]

L'intervention de l'État est donc double. Elle doit permettre le bon fonctionnement de l'économie de marché, elle doit faire émerger les fins différentes de l'économie qui sont nécessaires à la bonne conduite des affaires. De ce point de vue, l'économie comme science (Rougier n'hésite pas sur ce point épistémologique) ne peut pas décider des fins souhaitables assignées à une société. Elle peut juste évaluer les moyens économiques d'atteindre ces fins et leur « cohérence interne » [Rougier 1938b, 63-64]. Facteur déterminant de la cohésion sociale par sa capacité à produire tant du bien être que du bon ordre social, le marché n'en demeure pas moins qu'une des composantes d'une vie politique accomplie.

Cette composante reste centrale et s'articule à un destin fondamentalement démocratique. À la détermination d'une hiérarchie sociale par le jeu des compétences économiques, et à l'intégration de l'État et de son autorité dans le bon fonctionnement du marché, il va en effet ajouter un troisième terme : le peuple. Nous en avons déjà distingué deux grandes figures chez lui. Il y a le peuple honni, masse nationaliste, inculte, il y a le peuple réservoir d'élite. Une troisième figure, sous la forme bien particulière d'un paramètre économique, va s'imposer : le peuple comme ensemble de consommateurs. Rougier est peut-être à ce titre le premier à avoir donné une vision positive du « citoyen-consommateur », terme qu'il n'emploie pas, mais qui est la contrepartie d'une expression qu'il utilise volontiers notamment dans Les mystiques économiques : la démocratie des consommateurs, qui «exprime le plébiscite des prix sur les marchés libres » [Rougier 1938b, 80].

In fine, ce qui assure le succès du capitalisme comme force de cohésion sociale, c'est que son utilité est apparente à tous, par l'augmentation gé- 
nérale du niveau de vie. Mais cela, note-t-il, est un «problème technique, un problème d'équilibre économique, et de rendement » qui ne demande pas la remise en cause de l'ordre politique [Rougier 1929b, 48]. Chacun peut bénéficier d'une concurrence réglée, qui garantit les prix les plus bas, ce qui suppose de désencadrer le commerce, d'abaisser les frontières douanières, d'en finir avec le protectionnisme (non sans avoir conscience que le marché mondial étant imparfait, le protectionnisme doit se maintenir partiellement). La démocratie des consommateurs, c'est donc la capacité de fournir à tous des biens abordables, en plus grand nombre, et de meilleure qualité. D'où une fonction intégratrice du marché dont il donne un exemple frappant. Si l'on se souciait de faire vivre une économie de marché dynamique, qui permettrait aux consommateurs un accès élargi aux biens économiques par le biais de la concurrence, on favoriserait les ménages. Or, note-t-il, les ménages sont tenus par des ménagères [Rougier 1938b, 88]. La démocratie des consommateurs implique donc le statut prééminent des consommatrices. Sur la base du marché, la question du vote des femmes, qui fut si longtemps repoussée par la III ${ }^{\mathrm{e}}$ République trouve sa solution immédiate. Ici Rougier montre que le marché permet d'étendre pour des raisons économiques le corps politique sans révolution - pas de coup de force législatif, on reconnaît juste que les femmes sont des consommateurs - et sans même de réorganisation sociale : la femme dont il s'agit est bien la ménagère, au foyer, la femme traditionnelle. Ainsi, le marché devient pourvoyeur d'un bien politique fondamentalement démocratique (l'extension du droit de vote) par un simple équilibrage spontané. Il devient alors un facteur de cohésion politique et démocratique.

\section{***}

La philosophie de Rougier doit se considérer sur trois plans. Il y a les textes, nombreux, foisonnants, la réputation, parfois douteuse, et la force de la pensée. Les textes nous montrent un auteur qui n'a pas renoncé à l'ambition de savoir universel, qui peut apparaître, faute de s'expliciter, comme un intérêt pour des sujets très divers, et parfois fort hétéroclites. L'accès en est d'autant plus complexe que Rougier se distribue sur plusieurs registres d'intervention. Selon les textes qu'on abordera, nous verrons un essayiste brillant, polémique contre de Gaulle, un philosophe spécialisé (quand il s'agit de théorie des sciences ou d'économie), ou encore un graphomane des religions. La réputation est un pur phénomène sociologique. Quand on n'est ni un démocrate chrétien, ni un gaulliste, mais qu'on demeure un conservateur, l'appel de l'extrême droite n'est pas loin — une extrême droite intellectuelle et critique. Cela ne doit 
pas être confondu avec la récupération pure et simple dont Rougier a pu être victime de la part d'une extrême droite fort peu intellectuelle, et très dogmatique. Dénoncer les dérives où l'antigaullisme de Rougier l'entraîna, ses amitiés après la guerre, est une position politique, non une attitude philosophique. Mais c'est une attitude politique légitime, dont on peut juste demander qu'elle soit appliquée également à tous. Ni politiquement, ni moralement, Rougier ne ressemble aux cas célèbres d'un Heidegger ou d'un Carl Schmitt. En faire un collaborateur - ce qu'il ne fut jamais - ou un antisémite - ce que tout prouve qu'il ne fut en aucun cas, jusqu'à récuser tout soupçon en ce sens - est tout simplement inacceptable.

Reste la force de la pensée, et elle est indéniable. Quand on lit Rougier, on ne peut manquer de le plaindre d'avoir été français. Sans la guerre, sans Vichy, il serait simplement l'un des grands penseurs libéraux aux côtés de Hayek, ou Schumpeter, avec une hauteur de vue surprenante. On le verrait même un peu en avance de son temps, quand il s'agit de questions juridiques. Mais on ne saurait négliger, chez ce grand contemporain, l'homme d'histoire qui n'hésitait pas à rapprocher auteurs anciens et questions contemporaines ; à confronter le puritanisme protestant qui valorise le succès matériel, descendant, dit-il dans une phrase dure, « bien en dessous de l'hypocrisie » et la Bible qui invente, selon lui, l'accusation contre l'accumulation matérielle, vante la pauvreté, promet la revanche au misérable. Hérésie historique - même s'il se targue de rapporter à l'histoire ses conceptions - mais vérité philosophique? La réalité est plus complexe. Aux yeux de Rougier, cette démarche est une méthode d'appréhension des questions qui se posent parce qu'aucun problème philosophique n'est isolé d'une histoire, et que la confrontation des questions qui se posent est aussi bien la rencontre d'histoires qui se tissent. Ainsi Rougier nous offre une méthodologie pour saisir l'histoire des idées autrement que dans la boîte noire du passé. Le thème de la mystique, récurrent dans son œuvre nous explique en effet que les philosophies ne se donnent pas comme telles mais se sédimentent en des croyances dégagée de la contrainte d'argumentation qui initialement pèse sur elles. Inversons donc notre première position sur les questions mal posées : je disais qu'elles sont le Cain de ces Abel que sont les questions correctement formulées. Rougier nous montre que le destin d'une philosophie, quand elle réussit, est de se dégrader en une série de telles questions, parce qu'elle n'est plus même de l'ordre de l'interrogation. Cain n'est autre qu'Abel, projeté dans l'histoire. 